\title{
Characterization of a Quarter Torus Filter for Vacuum Arc Ion Flux Transmission
}

\author{
H. Kelly, A. Márquez*, and M. Pirrera \\ Instituto de Física del Plasma (CONICET), Departamento de Física, \\ Facultad de Ciencias Exactas y Naturales (UBA) \\ Ciudad Universitaria Pab. I, (1428) Buenos Aires, Argentina
}

Received on 19 December, 2003; revised version received on 30 April, 2004

\begin{abstract}
Measurements on the ion flux transmission in a magnetically filtered d-c vacuum arc are presented. The device includes a metallic plasma-generating chamber with water-cooled electrodes coupled to a substrate chamber through a quarter-torus magnetic filter. It is employed a Copper cathode $(6 \mathrm{~cm}$ in diameter) with a Copper annular anode. The filter consists in a steel cylinder $500 \mathrm{~mm}$ length and $100 \mathrm{~mm}$ inner diameter with $90^{\circ}$ of bending angle and corrugated lateral walls, surrounded by a magnetic field generating coil. The arc is operated at a current level of $100 \mathrm{~A}$, and the intensity of the filtering magnetic field was in the range $0-200 \mathrm{G}$ (measured at the knee of the filter). This magnetic field is enough high so as to magnetize the electrons but not the ions. The discharge is ignited by bringing (and later removing) a tungsten striker into contact with the cathode. The arc voltage drop, the floating potentials of the filter and the plasma, and the ion current collected by probes located at different positions as functions of the magnetic field intensity are reported and compared with measurements presented in the literature with other similar devices.
\end{abstract}

\section{Introduction}

The cathodic vacuum arc is widely used to produce coatings[1]. The deposited material comes from highly ionized plasma ejected from minute sizes on the cathode surface, known as cathode spots. The kinetic energies of the ions are in the range $15-120 \mathrm{eV}$, depending on the cathode material and on the charge-state of the ion[2,3], and with a total ion current amounting to $8-10 \%$ of the total discharge current[4].

The presence of microdroplets of melted cathode material in the coatings is a disadvantage in vacuum arc technology, since for some applications this macrodroplets increase the porosity and roughness of the coating. Several attempts have been made to separate the metallic plasma from the microdroplets by means of different filtering systems. The most popular of these systems are based on a focusing magnetic field that magnetize the electrons and guide the metallic plasma through the filter to the substrate but leave unchanged the microdroplet flux [5]. Unfortunately, part of the plasma flux is lost in the filter; so many efforts have been devoted to the optimization of filters that is removing all the microdroplets with the minimum losses of plasma. Straight $[6,7,8]$ and curved[5, 9, 10] filters have been studied. Presently, the one most often employed in practice is the so-called "quarter torus filter" (developed by Aksenov). It consists in a circular non-magnetic metallic tube with a bending angle of $90^{\circ}$, and with a toroidal magnetic field generated by an external coil. The magnetic field intensity is in the range $50-500 \mathrm{G}$, a value enough high to magnetize the plasma electrons. One of the most important findings in a quarter torus filter optimization consisted in applying a positive bias voltage (with respect to the plasma potential) of about $10-20 \mathrm{~V}$ to the filter.

In this work we present the first measurements performed with a magnetically filtered arc at INFIP (DCF2 device). The arc voltage drop and the floating potential of the filter as functions of the magnetic field intensity are reported. By employing also ion collectors, measurements of the ion flux transmission and plasma floating potential for different biasing voltages of the filter are reported and discussed.

\section{Experimental setup}

The investigations were carried out in a d-c filtered vacuum arc system, which is shown schematically in Fig. 1. There is a plasma generation chamber that includes a water-cooled copper cylindrical cathode (60 $\mathrm{mm}$ in diameter) surrounded by a floating shield, an annular water-cooler cooper anode ( $80 \mathrm{~mm}$ in diameter), and a tungsten striker which is brought into contact with the cathode surface and later removed to trigger the discharge. Both electrodes are mounted on an insulating piece that set an electrode separation of about 15 $\mathrm{mm}$. The anode was grounded. At the exit of this chamber is connected a magnetic quarter torus filter $(500 \mathrm{~mm}$ length, $100 \mathrm{~mm}$ inner diameter) made of corrugated stainless steel, including an external coil which produces the filtering magnetic field. The bending angle of the torus is $90^{\circ}$.

${ }^{*}$ Member of the CONICET 
At the exit of the torus a deposition stainless steel vacuum chamber (cross shaped) is connected. The electrodes system, magnetic filter and deposition chamber are electrically isolated among them. By employing an independent d-c power source, the magnetic filter can be biased with respect to the anode. Two vacuum systems (composed of mechanical and diffusion pumps) pump separately the plasma generation and deposition chambers to a base pressure of less than $10^{-4}$ mbar. The discharge circuit consisted in a current supply $(18 \mathrm{~kW}, 150 \mathrm{~A})$ in parallel with a capacitor bank $(165 \mathrm{mF})$ connected to the electrodes through a series inductor $(2.8 \mathrm{mH})$ in order to provide arc stability. The arc was operated in a continuous mode with an arc current of $100 \mathrm{~A}$.

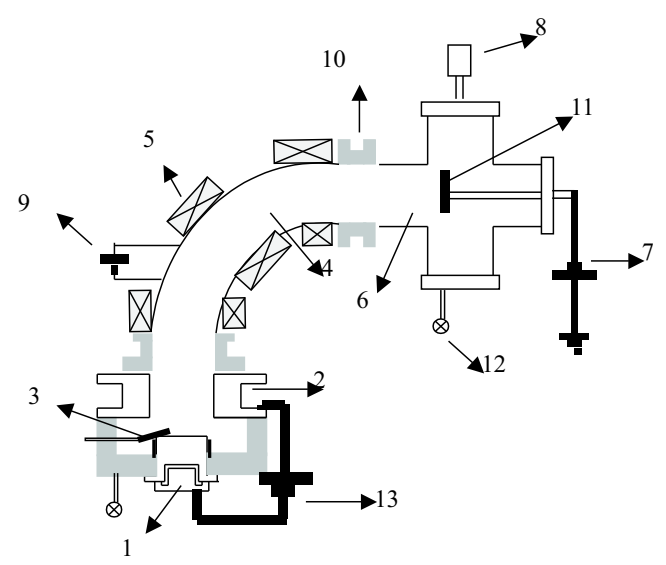

Figure 1. Scheme of the DCF2 device. (1) cathode; (2) anode; (3) trigger; (4) quarter torus magnetic filter; (5) torus coil; (6) deposition chamber; (7) probe bias source; (8) diagnostic port; (9) filter bias source; (10) insulators; (11) collecting probe; (12) vacuum pumping systems; (13) arc source.

The magnetic field generating coil was fed by an independent d-c variable current source, so that magnetic field values (measured by a Hall probe) varied in the range $0-197$ $\mathrm{G}$. The maximum field corresponded to a coil current of 55.2 $\mathrm{A}$, and was obtained at the knee of the torus.

The anode-cathode voltage drop was measured using a high impedance resistive voltage divider. The ion current at the exit of the filter was sensed introducing different sized collecting probes (probe A with a collecting area of $57 \mathrm{~cm}^{2}$, and probe B with a collecting area of $7.6 \mathrm{~cm}^{2}$ ) at different positions in the deposition chamber. The probes were biased at different voltages by a d-c power source, and the collected current was registered by measuring the induced voltage drop on a resistor connected in series with the biasing power source. Two high-impedance resistive voltage dividers were also employed to register the floating potentials of both the collecting probe and the filter. The electrical signals were registered in a four channel digitizing oscilloscope (sampling rate of $250 \mathrm{Ms} / \mathrm{s}$, analogical bandwidth of $500 \mathrm{MHz}$ ).

\section{Results}

In Fig. 2 a typical magnetic field $(B)$ profile is shown. The independent variable is the azimuthal angle of the torus $\phi$, measured with respect to its center of curvature $\left(\phi=0^{\circ}\right.$ coincides with the filter entrance, $\phi=45^{\circ}$ coincides with the filter knee and $\phi=90^{\circ}$ coincides with the filter exit). The coil current in Fig. 2 was $33 \mathrm{~A}$, given a maximum field at the knee $\left(B_{k}\right)$ of $120 \mathrm{G}$. It can be seen that the magnetic field is strongly inhomogeneous, and it drops at $\sim 10 \%$ of its maximum value $\left(B_{k}\right)$ at the filter exit. Other coil currents gave similar shaped $B$ profiles, since $B$ is proportional to the coil current. In what follows, we will use the maximum value $B_{k}$ to characterize the magnetic state of the filter.

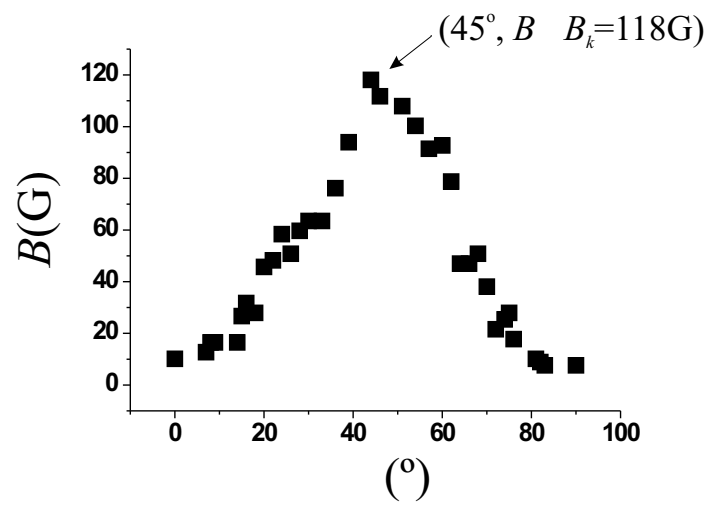

Figure 2. Magnetic field profile as a function of the azimuthal angle $\phi$, for a coil current of $33 \mathrm{~A}$; the maximum value of $B$ (at the knee of the quarter torus) is $B_{k}=118 \mathrm{G}$.

The arc voltage drop resulted independent of the magnetic field value and the filter bias operating conditions. For an arc current of $100 \mathrm{~A}$ it took a value of $(20 \pm 2) \mathrm{V}$.

The floating potentials (with respect to the grounded anode) of the magnetic filter $\mathrm{V}_{f f}$ and the probe $\mathrm{V}_{f p}$ as functions of $B_{k}$, are shown in Fig. 3. The probe was located at $20 \mathrm{~cm}$ of the filter exit. Each point in the figure represents an average of $3-5$ arc discharges performed under identical conditions. An individual measurement had a typical uncertainty of $10 \%$, due to the inherent noisiness of the arc plasma. It can be seen from Fig. 3 that $\mathrm{V}_{f f}<0$ for $B_{k}<75$ $\mathrm{G}\left(\mathrm{V}_{f f}\left(B_{k}=0\right)=-7 \mathrm{~V}\right)$ while it becomes positive for higher values of $B_{k}$. Since the plasma potential is quite close to the anode potential, a positive value for $\mathrm{V}_{f f}$ indicates a strong magnetization of the electrons. On the other hand, $\mathrm{V}_{f p}$ is always negative, and shows a complex behavior with $B_{k}$, presenting a kind of "potential well". In fact, $\mathrm{V}_{f p}$ varies from $-12 \mathrm{~V}$ at $B_{k}=0$ to $-40 \mathrm{~V}$ at $B_{k} \sim 30 \mathrm{G}$, and then slowly increases with $B_{k}$, reaching the value $\mathrm{V}_{f p}\left(B_{k}=197\right.$ $\mathrm{G})=-22 \mathrm{~V}$. It should be emphasized that the actual $B$ value at the probe position $\left(B^{*}\right)$ is in this experiment much smaller than the conventional $B_{k}$ value at the torus knee. For this reason, both values of $B\left(B_{k}\right.$ and $\left.B^{*}\right)$ have been plotted in the abscissa axis of Fig. 3. 


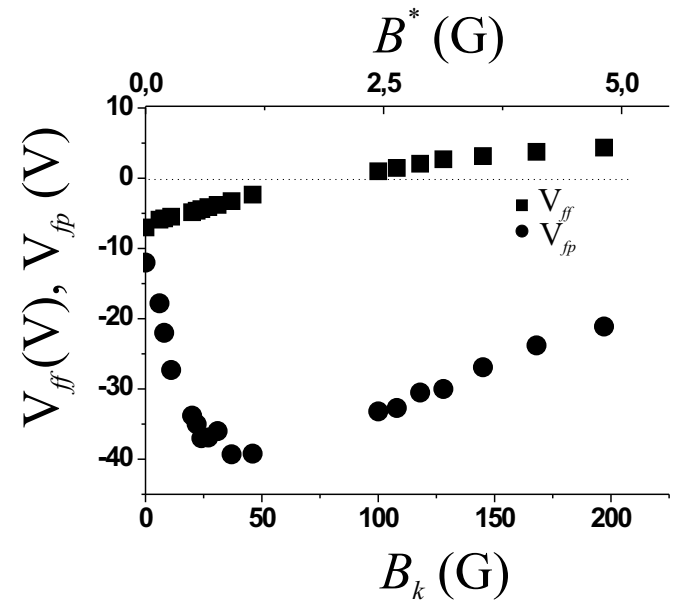

Figure 3. $\mathrm{V}_{f f}$ and $\mathrm{V}_{f p}$ as functions of the magnetic field at the filter knee $B_{k}$ (or equivalently, the magnetic field at the filter exit $B^{*}$ ) with the probe located at $\mathrm{L}=20 \mathrm{~cm}$ from the filter exit.

To study the influence of the probe on the filter floating potential, $\mathrm{V}_{f f}$ was measured for different biasing voltages of the probe $\left(\mathrm{V}_{b p}\right)$. The results are presented in Fig. 4, where $\mathrm{V}_{f f}$ is plotted against $B_{k}$ for $\mathrm{V}_{b p}=-50 \mathrm{~V}, \mathrm{~V}_{b p}=-30 \mathrm{~V}$ and $\mathrm{V}_{b p}=\mathrm{V}_{f p}$. It can be seen from Fig. 4 that for small values of $B_{k}$ (when $\mathrm{V}_{f f} \leqslant 0$ ) there is no clear dependence of $\mathrm{V}_{f f}$ on $\mathrm{V}_{b p}$, but for high values of $B_{k}\left(B_{k}>120 \mathrm{G}\right) \mathrm{V}_{f f}$ slightly increases with the absolute value of $\mathrm{V}_{b p}$.

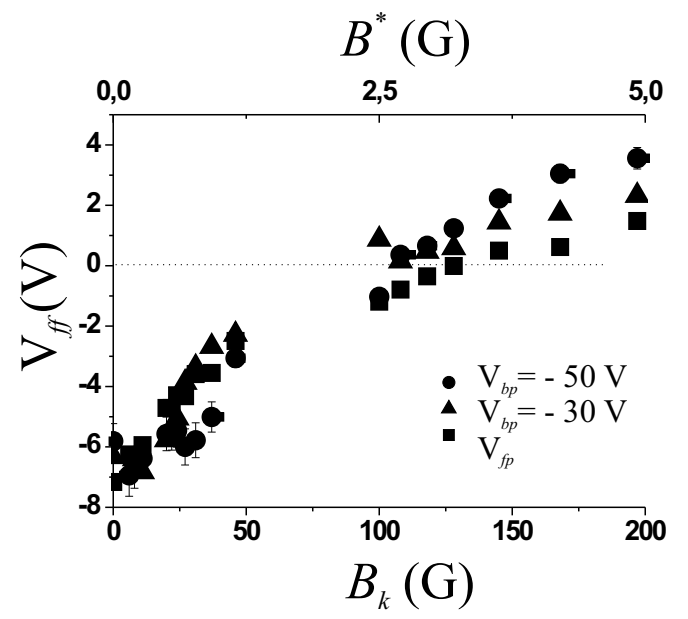

Figure $4 . \mathrm{V}_{f f}$ as a function of the magnetic field at the filter knee $B_{k}$ (or equivalently, the magnetic field at the filter exit $B^{*}$ ), at different probe voltages $\left(\mathrm{V}_{b p}\right.$ and $\left.\mathrm{V}_{f p}\right)$ for probe $\mathrm{A}$, with $\mathrm{L}=20 \mathrm{~cm}$.

In Fig. 5, the larger size probe current $\mathrm{I}_{p \mathrm{~A}}$ as a function of $B_{k}$, for different probe bias potentials $(-50 \mathrm{~V}$ and -30 $\mathrm{V})$ and floating filter is presented. For $\mathrm{V}_{b p}=-50 \mathrm{~V}, \mathrm{I}_{p \mathrm{~A}}$ is negative for low $B_{k}$ values, and increases with $B_{k}$, reaching positive values for $B_{k}>30 \mathrm{G}$. The situation for $\mathrm{V}_{b p}=-30$ $\mathrm{V}$ is quite similar to the previous one, but in this case $\mathrm{I}_{p \mathrm{~A}}$ reaches positive values for $B_{k}>140 \mathrm{G}$. The negative values of $\mathrm{I}_{p \mathrm{~A}}$ registered for low $B_{k}$ values are consistent with the high negative $\mathrm{V}_{f p}$ values shown in Fig. 3 .

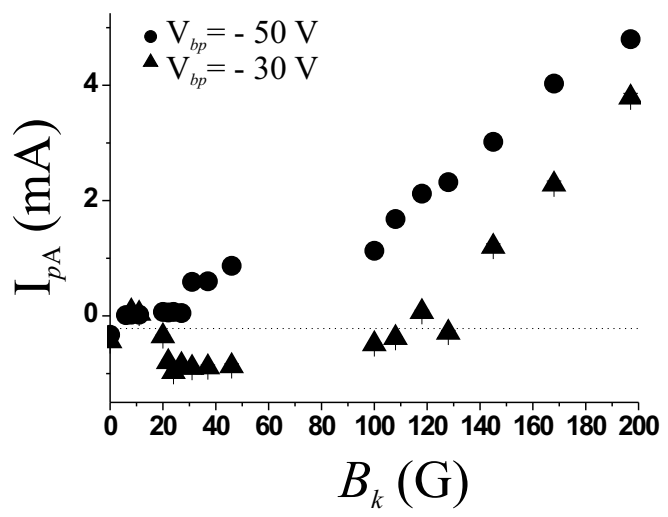

Figure 5. Probe current $\mathrm{I}_{p \mathrm{~A}}$ for floating filter as a function of the magnetic field at the filter knee $B_{k}$ at different $\mathrm{V}_{b p}$ values, with $\mathrm{L}$ $=20 \mathrm{~cm}$.

A comparison between the ion current collected by probes of different sizes is presented in Fig. 6. Both probes were located at $20 \mathrm{~cm}$ from the duct exit, biased at -50 $\mathrm{V}$ and with floating filter. The quotient between both currents coincides approximately with the quotient between the probe's areas, indicating a good homogeneity of the plasma at the probe's position.

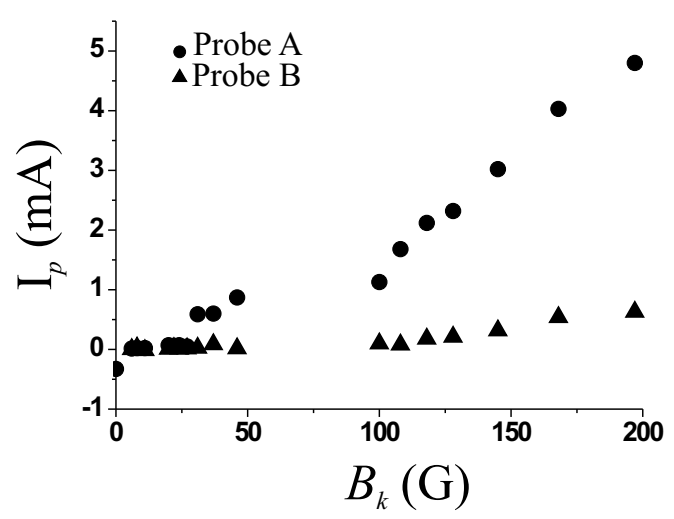

Figure 6. Probe current for floating filter as a function of the magnetic field at the filter knee $B_{k}$, with different probe sizes, $\mathrm{V}_{b p}=$ $-50 \mathrm{~V}$ and $\mathrm{L}=20 \mathrm{~cm}$.

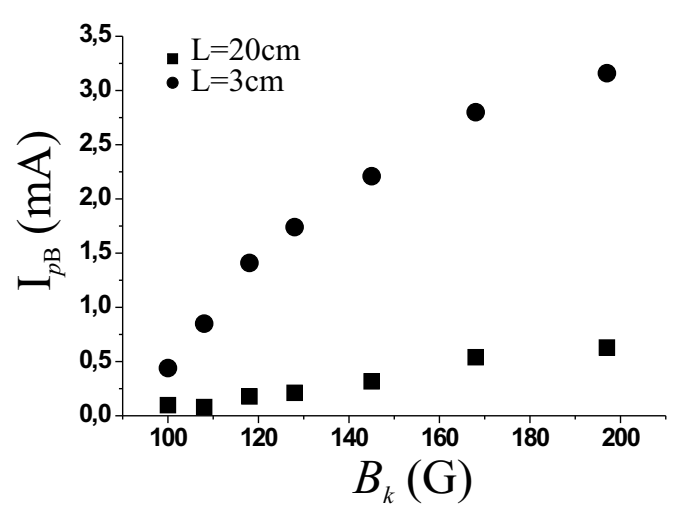

Figure 7. Probe current $I_{p \text { B }}$ for floating filter as a function of the magnetic field at the filter knee $B_{k}$ at different positions from the filter exit $(\mathrm{L}=3 \mathrm{~cm}$ and $20 \mathrm{~cm})$; with $\mathrm{V}_{b p}=-50 \mathrm{~V}$. 
In order to investigate the plasma expansion at the filter's exit, $\mathrm{I}_{p \mathrm{~B}}$ was registered at different distances from the filter. This is shown in Fig. 7, where $\mathrm{I}_{p \mathrm{~B}}$ is plotted as a function of $B_{k}$ for $\mathrm{V}_{b p}=-50 \mathrm{~V}$, floating filter, and $\mathrm{L}=3$ and $20 \mathrm{~cm}$, being $\mathrm{L}$ the axial distance between probe and filter's exit. It can be seen that the shorter distance results in an increased $\mathrm{I}_{p \mathrm{~B}}$ value (by a factor of $\sim 6$ ). For $B_{k}=197 \mathrm{G}$, the magnetic fields at both probe locations are: $B^{*}(\mathrm{~L}=3 \mathrm{~cm})=30 \mathrm{G}$ and $B^{*}(\mathrm{~L}=20 \mathrm{~cm})=5 \mathrm{G}$.

Figure 8 shows the curve $\mathrm{I}_{p \mathrm{~A}}$ vs. $B_{k}$ for different filter bias voltages $\left(\mathrm{V}_{b f}\right)$, for $\mathrm{V}_{p}=-50 \mathrm{~V}$. Each point in Fig. 8 represents the average of several runs (typically $3-5$ ) performed under identical values of $\mathrm{V}_{b f}$ and $B_{k}$. In practice, the value of $\mathrm{V}_{b f}$ could not be fixed "a priori" because the electron current collected by the filter produced a considerable voltage drop in the inner resistance of the biasing power source, thus resulting in a true $\mathrm{V}_{b f}$ equal to the prescribed biasing voltage minus the resistive source voltage drop. For arcs performed under the same nominal biasing filter voltages, the above-described effect resulted in the obtainment of a range of $\mathrm{V}_{b f}$ values (typically with an uncertainty of \pm 0.5 $\mathrm{V})$. It can be seen that $\mathrm{I}_{p \mathrm{~A}}$ increases with $B_{k}$. For low values of $B_{k}\left(B_{k}<85 \mathrm{G}\right)$ negative values of $\mathrm{I}_{p \mathrm{~A}}$ are found for $\mathrm{V}_{b f}$ values close to the filter floating potential (see Fig. 3). Note that the maximum $\mathrm{V}_{b f}$ value in Fig. 8 is $\mathrm{V}_{b f}=6 \mathrm{~V}$. This is due to the fact that the filter, when biased positively, collects a considerable negative current from the main arc discharge. In practice, with the presently available bias source, it was not possible to raise the bias voltage beyond $6 \mathrm{~V}$, because the biasing power source of the filter cannot withstand a current larger than $\approx 20 \mathrm{~A}$. The amount of this current is strongly dependent on $\mathrm{V}_{b f}$ (see next Figure).

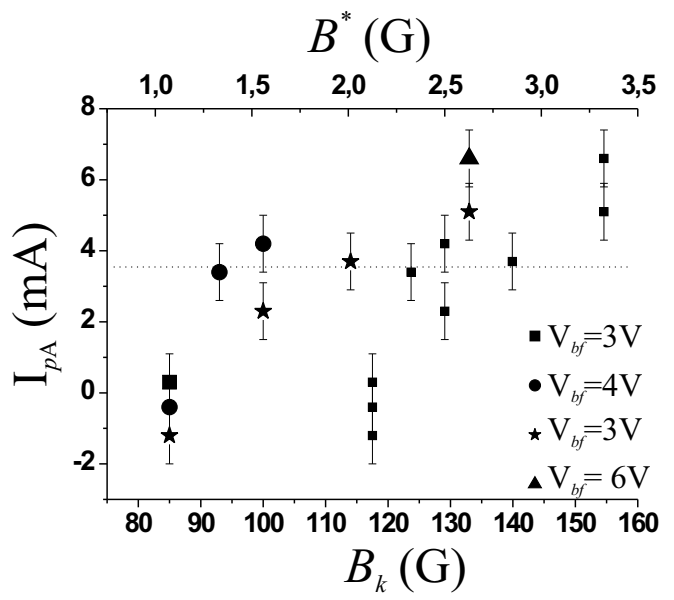

Figure 8. Probe current $\mathrm{I}_{p \mathrm{~A}}$ as a function of the magnetic field at the filter knee $B_{k}$ (equivalently, the magnetic field at the filter exit $\left.B^{*}\right)$ at different $\mathrm{V}_{b f}$ values and $\mathrm{V}_{b p}=-50 \mathrm{~V}$, with $\mathrm{L}=20 \mathrm{~cm}$.

Figure 9 shows the probe current $\mathrm{I}_{p \mathrm{~A}}$ and the current collected by the filter $\mathrm{I}_{f}$, as functions of $\mathrm{V}_{b f}$ for $B_{k}=154 \mathrm{G}$ and $\mathrm{V}_{b p}=-50 \mathrm{~V}$. Each point in the figure represents the average of several runs (typically $3-5$ ) performed under identical values of the nominal bias voltage. Note that $\mathrm{I}_{f}$ is negative (which means an electron current) and strongly increases (in absolute value) with $\mathrm{V}_{b f}$, indicating that the filter is acting as a secondary anode for the main discharge.
For instance, for $\mathrm{V}_{b f}=4 \mathrm{~V}$ the filter collects $20 \%$ of the discharge current for $B_{k}=154 \mathrm{G}$.

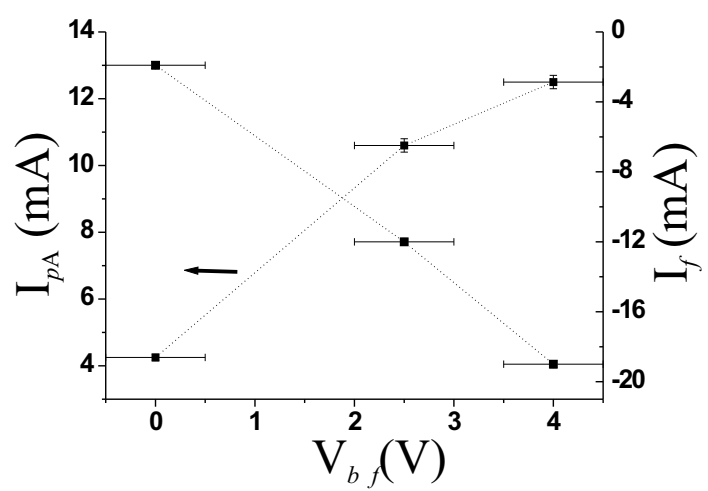

Figure 9. Probe current $\mathrm{I}_{p \mathrm{~A}}$ and filter current $\mathrm{I}_{f}$ as functions of the filter potential, with $\mathrm{V}_{b p}=-50 \mathrm{~V}, B_{k}=154 \mathrm{G}$ and $\mathrm{L}=20 \mathrm{~cm}$.

\section{Discussion and final remarks}

We have presented in this work the first measurements performed with a magnetically filtered arc at INFIP (DCF2 device). Although the optimization procedure is far from being complete (mostly due to the current limitation of the presently available filter bias source), the presented results show some interesting features.

The dependence of the filter floating potential with $B_{k}$ is in agreement with previous published results by other authors $[6,9]$, being negative for small $B_{k}$ values (when the electron flux reaching the filter still exceeds the ion flux) but becomes positive for high $B_{k}$ values (when the electrons are strongly magnetized, and cannot reach the filter). The behavior of the probe floating potential with $B_{k}$ presents some peculiarities never reported before, likely because of the small values of the magnetic field at the probe position $\left(B^{*}\right)$ at which these peculiarities are produced: the presence of a deep "floating potential well" $\left(\mathrm{V}_{f p} \sim-40 \mathrm{~V}\right.$, corresponding to $B^{*} \sim 1 \mathrm{G}$ ) when the electrons are partially magnetized (the electron Larmor radius is $\sim 3 \mathrm{~cm}$ for $B^{*} \sim 1$ $\mathrm{G}$ and an electron temperature $\mathrm{T}_{e} \sim 2 \mathrm{eV}$ ) can be explained only in terms of changes in the plasma potential in the presence of $B$, in the electron distribution function, and/or changes in probe charge collection theory. Note that this "potential well" has important practical consequences, because a probe must be biased at voltages well below $\mathrm{V}_{f p}$ to collect a pure ion current.

The value of $\mathrm{V}_{f p}$ at $B=0$ can be safely employed to determine $\mathrm{T}_{e}$, using classical electrostatic probe Langmuir theory corrected by Lam's work [11] to take into account ions of arbitrary kinetic energy. For $\mathrm{V}_{f p}=-12 \mathrm{~V}$, assuming that the plasma potential at $B=0$ is close to the anode ground potential, and using published vacuum arc data on average kinetic energy and charge-state for $\mathrm{Cu}$ ions (40 eV and 1.8, respectively) [12], it is obtained $\mathrm{T}_{e}=2.7 \mathrm{eV}$, in agreement with other published data for arcs operating in vacuum [12]. It is also worth noting that $\mathrm{V}_{f f}$ at $B=0$ cannot be used to determine $\mathrm{T}_{e}$, because probe theories cannot be used for an 
object of large size where the embedded plasma changes its properties.

The $\mathrm{Cu}$ ion currents collected by the probes correspond to plasma densities of $10^{8}-10^{9} \mathrm{~cm}^{-3}$ at the filter exit, depending on the operating arc conditions. These densities produce a Debye length (that is of the order of the thickness of the plasma sheath at the filter wall) in the range of $0.3-1$ $\mathrm{mm}$ for $\mathrm{T}_{e}=2.7 \mathrm{eV}$.

The best ion current collected at the filter exit amounts to $\approx 12 \mathrm{~mA}$ for $B_{k}=154 \mathrm{G}$, a value still small as compared with the maximum ion current $(\approx 8-10 \mathrm{~A})$ generated at the cathode surface for an arc discharge current of $100 \mathrm{~A}$. The optimum ion current was obtained at a filter bias voltage of $4 \mathrm{~V}$, when the filter, acting as a secondary anode, collected about $20 \%$ of the main discharge current. This result indicates that to obtain an improvement in the ion transmission through the filter, the first task is to get a good matching between the plasma generation chamber and the filter entrance, which will be done in the near future by generating a focusing magnetic field in the cathode-filter region so as to optimize the ion flux entering into the filter. Also, it is necessary to increase the filter bias voltage by employing a power source with a higher current capacity. The strong decrease in the ion current as the probe-filter distance increases (see Fig. 7) indicates that it is also necessary to increase the magnetic field at the probe location.

\section{Acknowledgments}

This work was supported by grants from the Universidad de Buenos Aires (PID X214) and from the Agencia Nacional de Promoción Científica y Tecnológica (PICT 03-09491).

\section{References}

[1] R. L. Boxman, D. M. Sanders, and P. J. Martin, Handbook of Vacuum Arc Science and Technology, Fundamentals and Applications, Park Rige, NJ: Noyes, 1995.

[2] I. G. Brown, B. Feinberg, and J. E. Galvin, J. Appl. Phys. 63, 4889 (1988).

[3] G. Y. Yushkov, A. Anders, E. M. Oks, and I. G. Brown, J. Appl. Phys. 88, 5618 (2000).

[4] C. W. Kimbling, J. Appl. Phys. 44, 3074 (1973).

[5] I. I. Aksenov, V. A. Belous, V. G. Padalka, and V. M. Khoroshikh, Sov. J. Plasma Phys. 4, 425 (1978).

[6] B. Cluggish, IEEE Trans. Plasma Sci. 36, 1645 (1998).

[7] V. N. Zhitomirsky, O. Zarchin, R. L. Boxman, and S. Goldsmith, Proc. 20th Int. Symp. on Discharges and Electrical Insulation in Vacuum (Tours, France), 670 (2002).

[8] H. Kelly, L. Giuliani, and F. Rausch, J. Phys. D: Appl. Phys. 36, 1980 (2003).

[9] A. Anders and S. Anders, and I. Brown, Plasma Source Sci. Technol. 4, 1 (1995).

[10] R. L. Boxman, V. N. Zhitomirsky, B. Alterkop, E. Gidalevich, M. Keidar, and S. Goldsmith, Surf. Coat. Technol. 86-87, 243 (1996).

[11] S. H. Lam, Phys. Fluids 8, 73 (1965).

[12] S. M. Shkolnik, IEEE Trans. Plasma Sci. 13, 336 (1985). 